\title{
The family Crassulaceae in continental Portugal
}

\section{Gideon F. Smith ${ }^{1}$ and Estrela Figueiredo ${ }^{2}$}

${ }^{1}$ Office of the Chief Director: Biosystematics Research \& Biodiversity Collections, South African National Biodiversity Institute, Private Bag X101, Pretoria, 0001 South Africa / H.G.W.J. Schweickerdt Herbarium, Department of Plant Science, University of Pretoria, Pretoria, 0002 South Africa / Centre for Functional Ecology, Departamento de Ciências da Vida, Universidade de Coimbra, 3001-455 Coimbra, Portugal (email: g.smith@sanbi.org.za).

2 Department of Botany, P.O. Box 77000, Nelson Mandela Metropolitan University, Port Elizabeth, 6031 South Africa / Centre for Functional Ecology, Departamento de Ciências da Vida, Universidade de Coimbra, 3001-455 Coimbra, Portugal (email: estrelafigueiredo@hotmail.com).

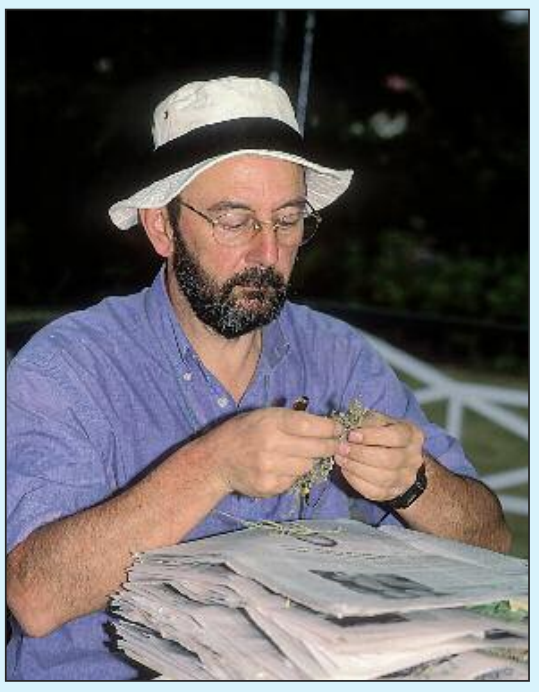

\section{Santiago Castroviejo}

This paper is respectfully dedicated to Dr Santiago Castroviejo Bolivar (b. 7 August 1946, d. 30 September 2009), who treated the Crassulaceae for the Flora iberica project, of which he was a major architect. He had a broad international participation in botanical thrusts of a global nature and his untimely death leaves a void that is felt beyond his native Spain.

\section{Photograph opposite}

Santiago Castroviejo processing collected material gathered around Ladysmith, KwaZulu-Natal province, during a field trip to South Africa in 1999. Photographer: A. Prunell.

Summary: Apart from two present-day centres of high species diversity, one in southern Africa and the other in Mexico, the Crassulaceae, a large family of mainly herbaceous leaf succulents, is also well-represented in Europe in terms of both genera and species. One of these genera, the predominantly continental European genus Sedum L., has for a long time been acknowledged as in need of being split into smaller, more homogenous genera. The recent implementation of some of these taxonomic proposals is here applied to the crassuloid taxa of continental Portugal (islands excluded), with particular reference to Petrosedum Grulich. An updated and expanded checklist of the genera and species of Crassulaceae occurring in Portugal is provided, along with notes on differences between Sedum and Petrosedum. Thirty-six species of Crassulaceae are now recorded for Portugal (continent, excluding islands), of which 14 are introduced.

Zusammenfassung: Abgesehen von zwei heutigen Zentren hoher Artdiversität (eines im südlichen Afrika, das andere in Mexiko), sind die Crassulaceae (eine grosse Familie krautiger Blattsukkulenten) in Bezug auf Gattungen wie Arten auch in Europa gut vertreten. Für eine dieser Gattungen, d.h. die vorwiegend kontinentaleuropäische Gattung Sedum L., war seit einiger Zeit die Notwendigkeit einer Aufteilung in kleinere, homogenere Gattungen klar. Die kürzliche Implementierung einiger dieser taxonomischen Vorschläge wird hier auf die Taxa des kontinentalen Portugals (ausgenommen Inseln) übertragen, mit besonderer Berücksichtigung von Petrosedum Grulich. Zudem wird eine aktual- 
isierte und erweiterte Checkliste der Gattungen und Arten der in Portugal vorkommenden Crassulaceae publiziert, zusammen mit Bemerkungen zu den Unterschieden zwischen Sedum und Petrosedum. Derzeit sind 36 Arten der Crassulaceae für Portugal (Kontinent, ohne Inseln) nachgewiesen, von welchen 14 eingeführt sind.

\section{Introduction}

The family Crassulaceae have a near cosmopolitan geographical distribution range, occurring on most continents, but with significant presentday centres of diversity in southern Africa (Smith et al., 1997) and Mexico (Thiede \& Eggli, 2007). Both intra- and inter-generic relationships in the family Crassulaceae have been the subjects of studies that resulted in considerable naming and renaming of species, facilitating more recent work to clarify the phylogeny of the family ('t Hart \& Eggli, 1995; Van Ham \& 't Hart, 1998; Mort et al., 2001).

In Europe, the Crassulaceae are represented by several genera, most of which consist of small, weedy, sub-shrublets (Webb et al., 1964; 't Hart \& Eggli, 1995). Sedum L. is the most speciose of the European genera, and has for a long time been considered to encompass entities that could be more comfortably accommodated in separate genera (Stephenson, 2007a). More recently, earlier suggestions by Berger (1930) that could better reflect crassuloid diversity at the generic rank were implemented through the general acceptance of the transferral of some species of Sedum to the genus Petrosedum Grulich (Grulich, 1984).

European species of Sedum can be roughly divided into four sections, each comprising similar, closely related species (Stephenson, 1994). Berger (1930) grouped one of these, the orthocarpic Sedum species with yellow or yellowish flowers, into Sedum ser. Rupestria. They have tall, densely flowered inflorescences with carpels that remain upright until they shed their seed, and are considered endemic to Europe or neighbouring areas of North Africa and the Middle East (Stephenson, 1994).

Grulich (1984) erected the new genus Petrosedum Grulich in which to accommodate species of Sedum ser. Rupestria, simultaneously publishing new combinations for $P$. forsterianum (Sm.) Grulich, P. pruinatum (Brot.) Grulich and P. sediforme (Jacq.) Grulich. Based on Grulich's grouping, Velayos (1989) later published the new combinations for P. amplexicaule (DC.) Velayos and $P$. amplexicaule subsp. tenuifolium (Sm.) Velayos.

In Flora iberica, Castroviejo \& Velayos (1997a) synonymised Petrosedum, and upheld Sedum ser.
Rupestria. Thiede \& Eggli (2007), on the other hand, accepted Petrosedum. This treatment is followed in the present paper.

This paper updates the generic concepts recently proposed for the Crassulaceae in Europe and provides an updated checklist of the species of the family that occur in continental Portugal, both naturally and as alien introductions (Castroviejo, 1997a; Sequeira et al., 2011). In addition, the four species of Petrosedum that occur in Portugal are briefly discussed and a key useful to separate them, especially in the vegetative phase, is provided. This paper also updates and supplements the treatment of Sedum as included in the Flora iberica.

\begin{tabular}{|l|c|}
\hline Genus & $\begin{array}{c}|c| \\
\text { Species } \\
\text { (introduced species) }\end{array}$ \\
\hline $\begin{array}{l}\text { *Aeonium Webb \& } \\
\text { Berthel. }\end{array}$ & $2(2)$ \\
\hline *Aichryson Webb \& \\
Berthel.
\end{tabular}

Table 1. Genera and number of species of Crassulaceae occurring in continental Portugal. (* = introduced genus) 


\section{Key to the species of Sedum and Petrosedum in Portugal}

1. Petals yellow (rarely creamy), anthers yellow

1. Petals white or pinkish, anthers red or purple

2. Growth form shrubby, up to $75 \mathrm{~cm}$ high Sedum praealtum*

2. Growth form herbaceous, much smaller 3

3. Sepals free; follicles spreading in a star-shape Sedum acre

3. Sepals united at the base; follicles erect 15 (Petrosedum)

4. Stamens $4-5$, as many as the sepals .5

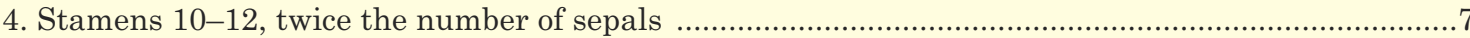

5. Plants glabrous 6

5. Plants glandulose Sedum rubens

6. Flowers pedicellate; follicles erect Sedum andegavense

6. Flowers sessile; follicles spreading in a star-shape Sedum caespitosum

7. Petals united at the base for $1 / 4-3 / 4$ of their length, forming a tube 8

7. Petals free .11

8. Corolla $>7 \mathrm{~mm}$ long Sedum mucizonia

8. Corolla $<7 \mathrm{~mm}$ long 9

9. Plants glabrous

Sedum candollei

9. Plants glandulose 10

10. Annual, in humid areas; without basal rosettes Sedum maireanum

10. Perennial, in rocky areas; stems arising from basal rosettes Sedum hirsutum

11. Sepals free; follicles spreading in a star-shape or sub-erect .12

11. Sepals united at the base; follicles erect 13

12. Annual, erect, stem branched from the base with ascending branches Sedum arenarium

12. Perennial, prostrate-ascending, multi-stemmed, radiating, much branched Sedum anglicum

13. Annual, with an erect stem, branched above Sedum pedicellatum

13. Perennial, caespitose, multi-stemmed, with sterile branches .14

14. Leaves of sterile branches alternate, ovoid to cylindrical; sepals obtuse Sedum album

14. Leaves of sterile branches opposite in 4 rows, subglobose; sepals acute Sedum brevifolium

\section{(Petrosedum)}

15. Leaves thick, succulent, apically flared open, boat-shaped in outline and cross-section; stems robust, $5 \mathrm{~mm}$ or more thick Petrosedum sediforme

Leaves thin, slightly succulent, hair-like, apically incurved; stems flimsy, 0.5-2.0 mm thick ......16 16. Leaf bases broadened, stem-clasping Petrosedum amplexicaule

Leaf bases joined to stem at a narrow point .17

17. Dried leaves on sterile branches shed early yielding a smooth, leafless stem, except at the apex where the leaves are clustered into a small ball-shaped rosette; plant green or glaucous

Petrosedum pruinatum

Dried leaves on sterile branches persistent yielding a leafy, club moss-like stem; plant reddish....... Petrosedum forsterianum 


\section{Genera and species of Crassulaceae in Por- tugal}

The Flora iberica project (Aedo, 2013), which aims to provide a comprehensive hard-copy and on-line identification and information tool to the flora of the Iberian Peninsula lists nine crassuloid genera as occurring on the subcontinent. Of these, seven were recorded for Portugal, comprising a total of 27 species of Crassulaceae recorded for this country. Sequeira et al. (2011) similarly list 27 species of Crassulaceae for continental Portugal.

A recent assessment of introduced plant species in Portugal (Almeida \& Freitas, 2006) resulted in two further genera (Graptopetalum Rose and Kalanchoe Adans.) being recorded for the country, in addition to the genera Petrosedum (see above), and Hylotelephium H.Ohba, which had been previously recorded but were omitted in Flora iberica (Table 1). The total number of species of Crassulaceae recorded for Portugal therefore increases to 36, of which 14 are introduced.

\section{Key to the species of Sedum and Petrosedum in Portugal}

The key to the genera of Crassulaceae in Flora iberica (Castroviejo, 1997a) does not include the genus Petrosedum. This genus can be separated from Sedum on account of a combination of characters. It consists of perennial plants with 5-12merous flowers, sepals united at the base, petals free, yellow, white or creamy, stamens 10-24, with yellow anthers, erect follicles, and oblong seeds. An artificial key to the 14 species of Sedum and 4 species of Petrosedum occurring naturally and as introduced aliens (*) in Portugal is provided on the opposite page.

\section{Checklist of crassuloid taxa occurring in Portugal.}

Synonyms are here restricted to those relevant to continental Portugal, and references pertain to the Portuguese flora. Synonyms, occurrence by province and vernacular names are based on Castroviejo (1997a). Province delimitations and abbreviations follow Aedo (2013) (see Figure 1).

1. *Aeonium Webb \& Berthel.

$1.1{ }^{*}$ A. arboreum (L.) Webb \& Berthel.; Santos Guerra pro parte (1997a: 117); Almeida \& Freitas (2006: 119); Sequeira et al. (2011).

= Sempervivum arboreum L.; Coutinho (1939: 335); Sampaio (1947: 375).

V.n.: Saião

BL E

Introduced from Macaronesia.

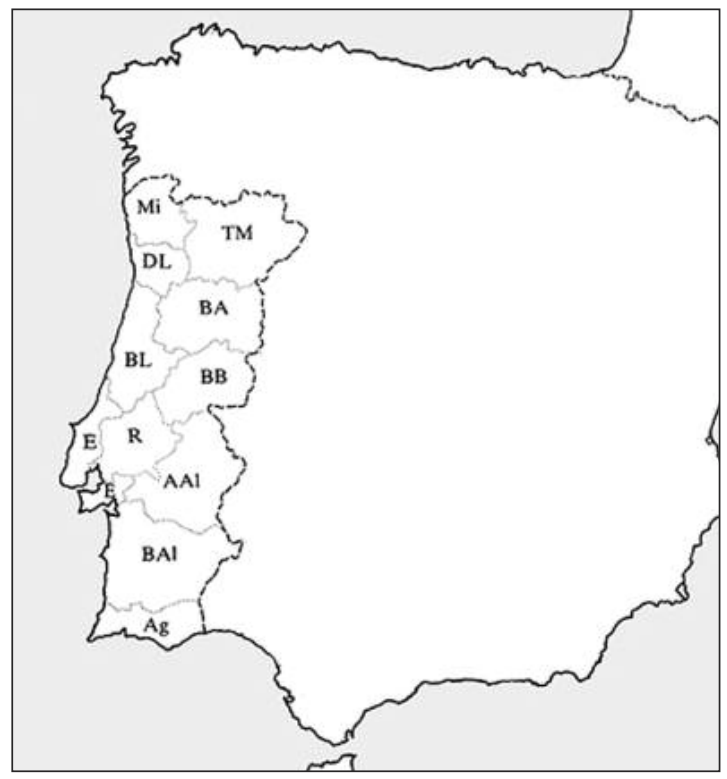

Figure 1. Map showing the delimitation of provinces in Portugal, based on Aedo (2013).

$1.2 *$ A. haworthii Salm-Dyck ex Webb \& Berthel.; Almeida \& Freitas (2006: 119).

Introduced from Macaronesia.

Note: Treated as a synonym of $A$. arboreum by Santos Guerra (1997a: 117), but here retained as a distinct species.

2. *Aichryson Webb \& Berthel.

2.1. *A. laxum (Haw.) Bramwell; Santos Guerra (1997b: 119); Nyffeler (2003: 25, 26); Almeida \& Freitas (2006: 119); Sequeira et al. (2011).

= A. dichotomum (DC.) Webb; Franco (1971: 248).

= Sempervivum annuum C.Sm. ex Buch.; Coutinho (1939: 335).

= S. dichotomum DC.; Sampaio (1947: 375).

Naturalized in the area of Sintra (E).

Introduced from the Canary Islands.

3. Crassula L.

3.1. ${ }^{*}$ C. aquatica (L.) Schönland; Franco (1971: 246); Fernandes (1997: 103); Almeida \& Freitas (2006: 121); Sequeira et al. (2011).

Naturalized in the area of Montemor-o-Velho (BL) Introduced from Eurasia and North America.

$3.2{ }^{*}$ C. multicava Lem.; Almeida \& Freitas (2006: 121).

Introduced from South Africa.

$3.3{ }^{*}$ C. muscosa L.; Almeida \& Freitas (2006: 121). Introduced from South Africa. 


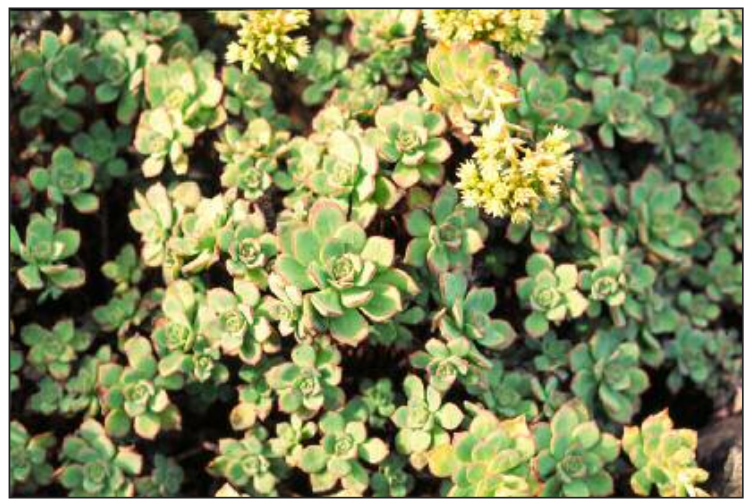

Figure 2. *Aeonium haworthii.

Photograph: Gideon F. Smith.

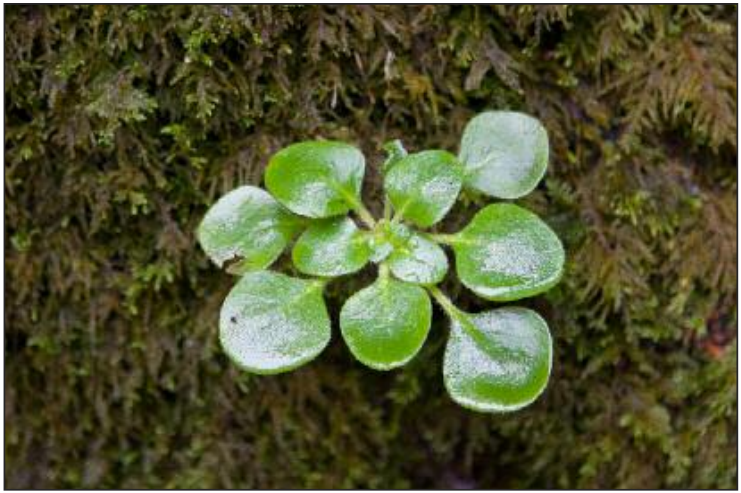

Figure 3. *Aichryson laxum. Photograph: M. Porto.

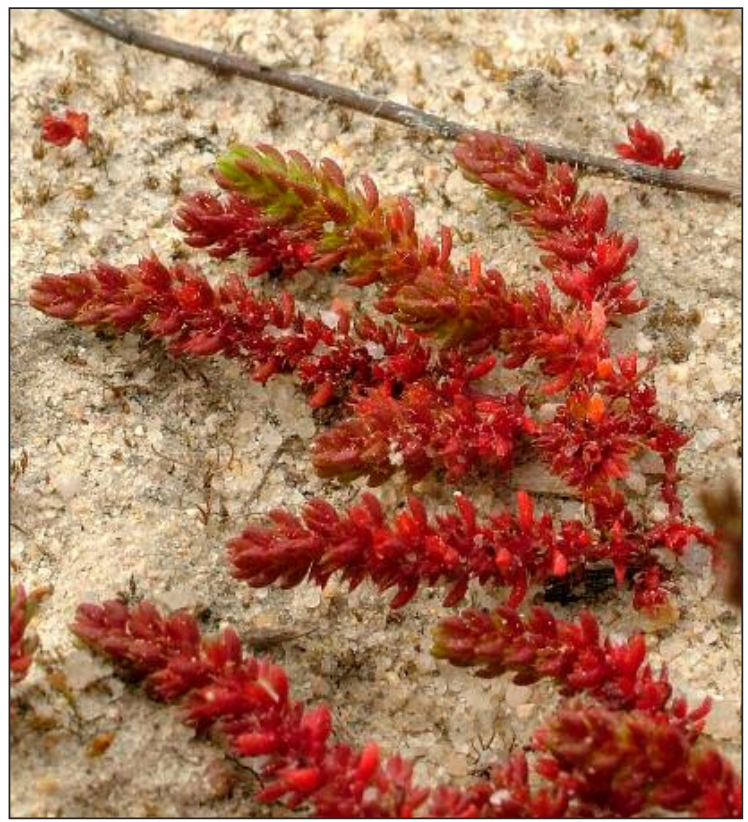

Figure 4. Crassula tillaea. Photograph: M. Porto.

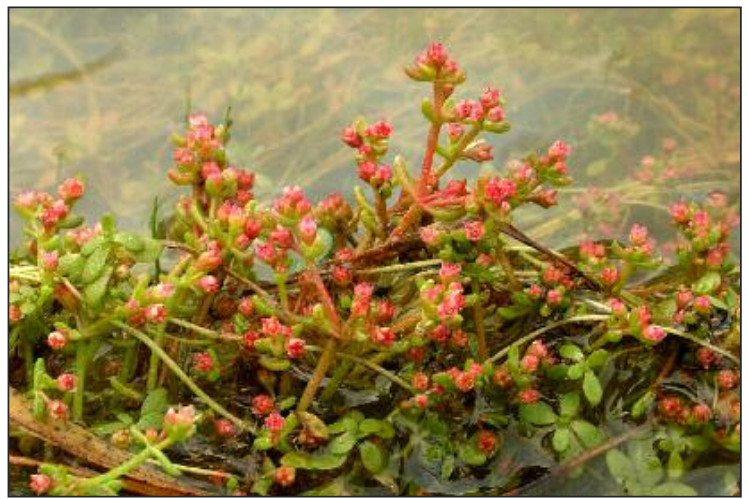

Figure 5. Crassula vaillantii.

Photograph: M. Porto.

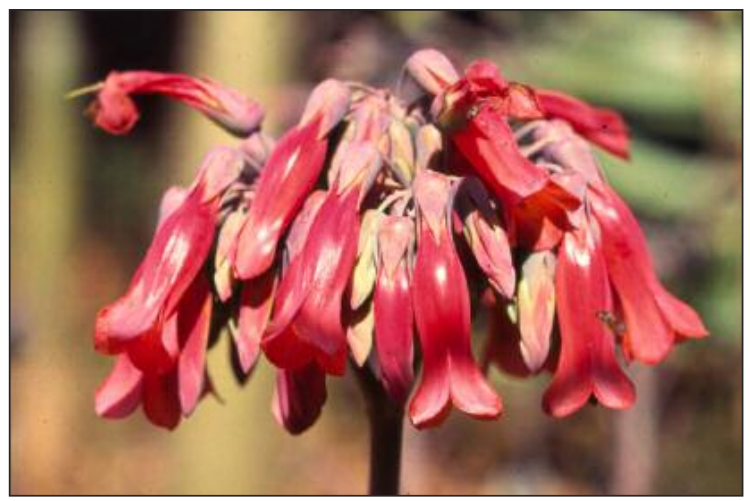

Figure 6. Inflorescence of *Kalanchoe delagoensis. Photograph: Gideon F. Smith.

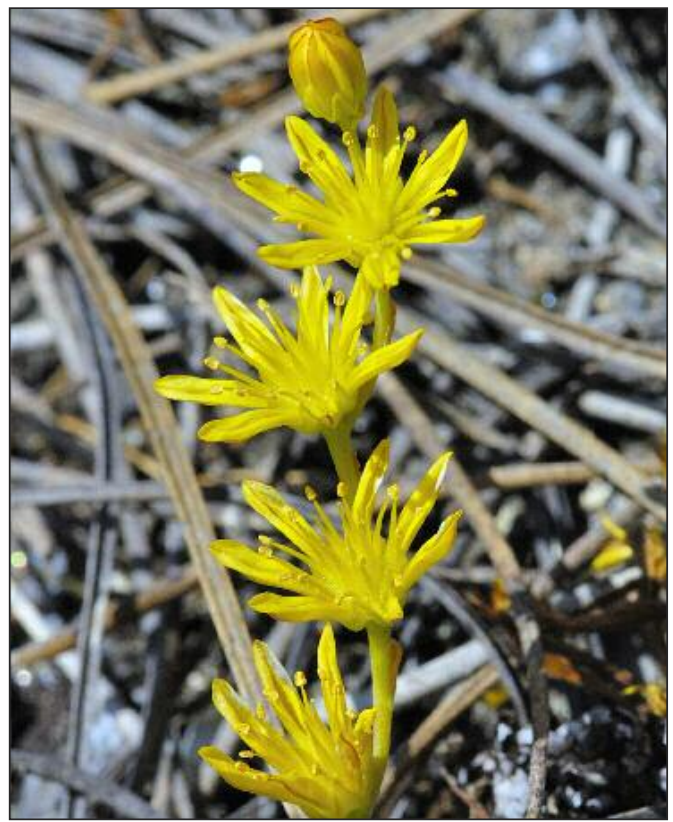

Figure 7. Petrosedum amplexicaule.

Photograph: P.V. Araújo. 


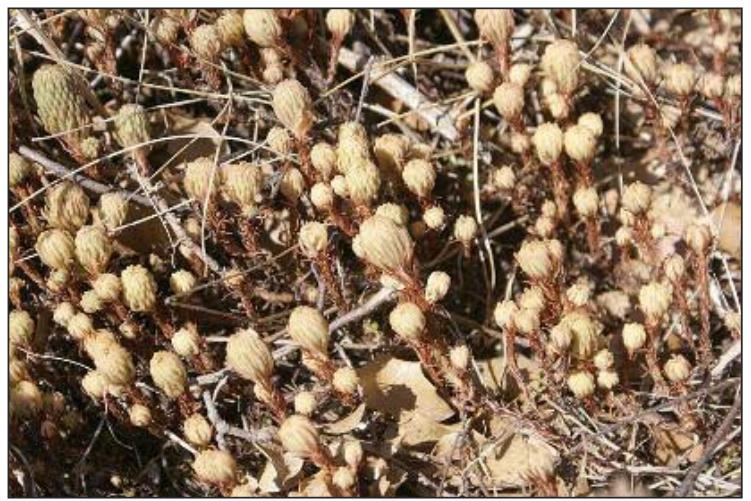

Figure 8. Petrosedum forsterianum.

Photograph: Gideon F. Smith.

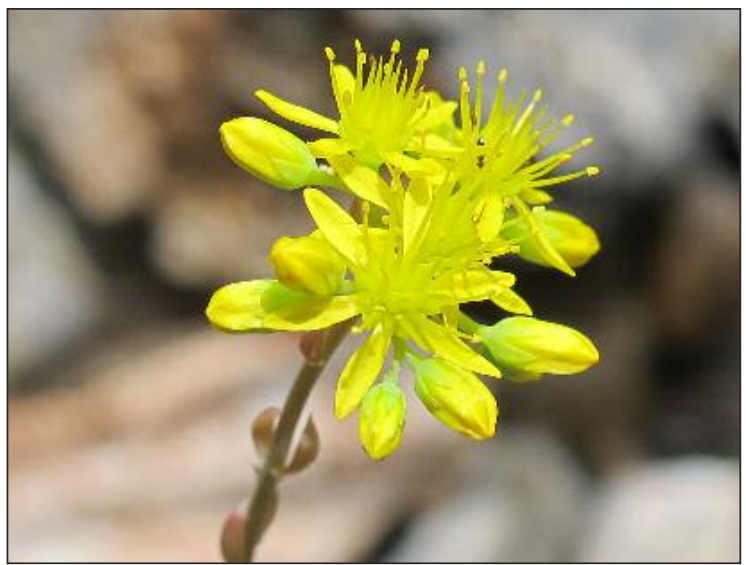

Figure 9. Petrosedum forsterianum.

Photograph: Estrela Figueiredo.

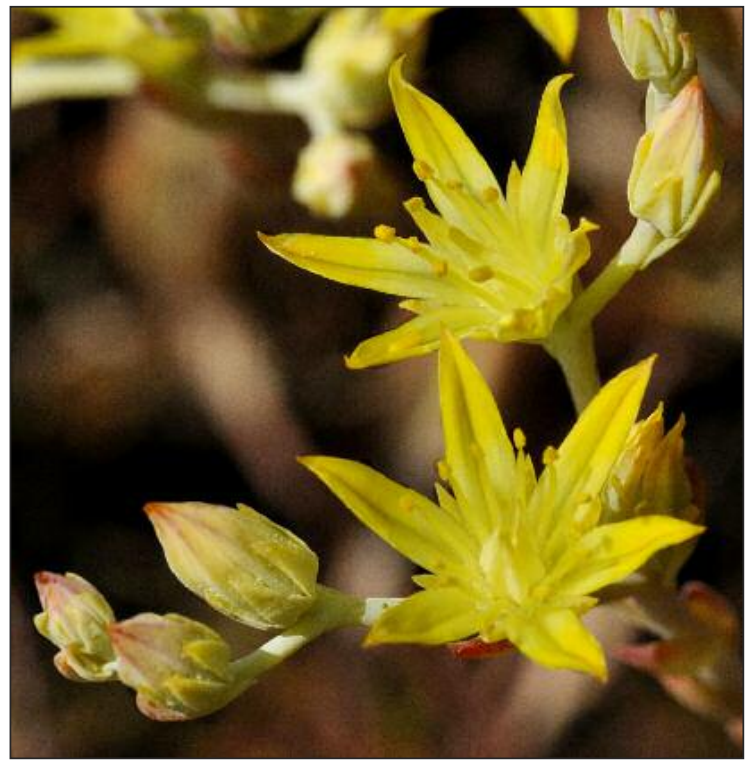

Figure 10. Petrosedum pruinatum.

Photograph: P.V. Araújo.

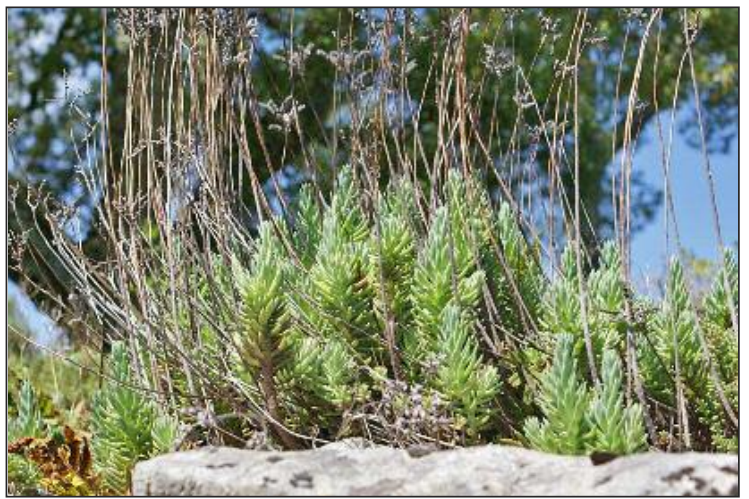

Figure 11. Petrosedum sediforme.

Photograph: Gideon F. Smith.

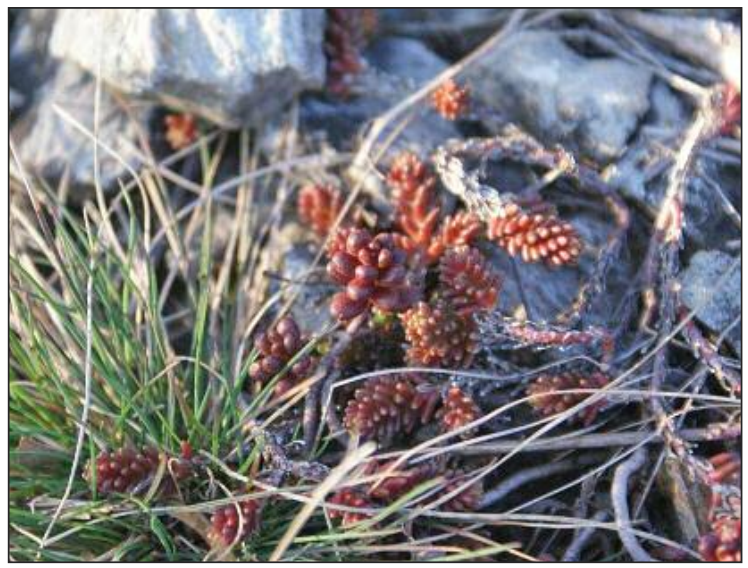

Figure 12. Sedum acre.

Photograph: Gideon F. Smith.

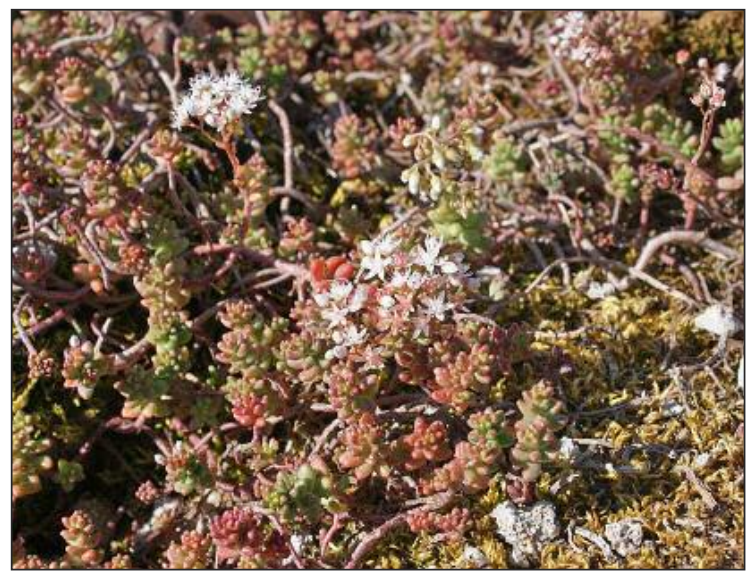

Figure 13. Sedum album in flower.

Photograph: Gideon F. Smith. 
3.4. ${ }^{*}$ C. peduncularis (Sm.) Medigen; Fernandes (1997: 103); Almeida \& Freitas (2006: 121); Sequeira et al. (2011).

$=$ C. bonariensis (DC.) Cambess.; Franco (1971: 246).

Naturalized in the area of Montemor-o-Velho (BL) Introduced from South America.

3.5. C. tillaea Lest.-Garl.; Franco (1971: 246); Fernandes (1997: 100); Eggli (2003b: 81); Sequeira et al. (2011).

= Tillaea muscosa L.; Coutinho (1939: 337); Sampaio (1947: 371).

Aal Ag BA Bal BB BL DL E Mi R TM

3.6. C. vaillantii (Willd.) Roth.; Franco (1971: 246); Fernandes (1997: 102); Van Jaarsveld (2003: 83); Sequeira et al. (2011).

= Tillaea vaillantii Willd.; Coutinho (1939: 337); Sampaio (1947: 371).

$\mathrm{AAl}$ Ag BAl DL R TM

4. *Graptopetalum Rose

$4.1{ }^{*} G$. paraguayense (N.E.Br.) E.Walther; Almeida \& Freitas (2006: 123).

Introduced from Mexico.

5. *Hylotelephium H.Ohba

5.1. *H. telephium (L.) H.Ohba

= Sedum complanatum Gilib., nom. illeg.; Coutinho (1939: 332).

V.n.: Fabária-maior, Erva-dos-calos.

Although Hylotelephium was not recorded for Portugal by Castroviejo \& Velayos (1997b), it was recorded earlier by Coutinho (1939) as naturalized in BA, E, and by Sampaio (1947) as subspontaneous. Sampaio (1947) recorded it under the name Sedum haematodes Mill. (= Hylotelephium hematodes (Mill.) Holub.), which leaves some doubt as to the exact identity of the record.

Introduced from $\mathrm{C}$ and $\mathrm{W}$ Europe.

6. *Kalanchoe Adans. [Some of the weedy species included in Kalanchoe are often referred to the genus Bryophyllum Salisb. See for example Crouch \& Smith (2007) and Walters (2011)]

$6.1{ }^{*} K$. daigremontiana Raym.-Hamet \& H.Perrier; Almeida \& Freitas (2006: 123).

Introduced from Madagascar.

6.2 * K. pinnata (Lam.) Pers.; Almeida \& Freitas (2006: 123).

Introduced from tropical Africa.

$6.3{ }^{*} K$. delagoensis Eckl. \& Zeyh.

= K. tubiflora (Harv.) Raym.-Hamet, nom. illeg.; Almeida \& Freitas (2006: 123).

Introduced from Madagascar.
7. Petrosedum Grulich

7.1. P. amplexicaule (DC.) Velayos

$=P$. amplexicaule subsp. tenuifolium (Sm.) Velayos

= Sedum amplexicaule DC.; Coutinho (1939: 333); Castroviejo \& Velayos (1997a: 142); Sequeira et al. (2011).

= S. tenuifolium (Sm.) Strobl; Sampaio (1947: 375); Franco (1971: 250).

AAl Ag BA BAl BB BL Mi R TM

7.2. P. forsterianum (Sm.) Grulich

= Sedum forsterianum $\mathrm{Sm}$.; Coutinho (1939: 333);

Sampaio (1947: 374); Franco (1971: 250); Castroviejo \& Velayos (1997a: 144).

V.n.: Arroz-das-paredes

AAl Ag BA BAl BB BL DL E Mi R TM

7.3. P. pruinatum (Brot.) Grulich

= Sedum pruinatum Brot.; Coutinho (1939: 333);

Sampaio (1947: 375); Franco (1971: 250); Castroviejo \& Velayos (1997a: 145); Sequeira et al. (2011).

BA BB DL Mi TM

7.4. P. sediforme (Jacq.) Grulich; Smith \& Figueiredo (2010b).

= Sedum altissimum Poir.; Coutinho (1939: 333).

$=$ S. nicaeense All.; Sampaio (1947: 375).

= S. sediforme (Jacq.) Pau; Franco (1971: 250); Castroviejo \& Velayos (1997a: 148); Sequeira et al. (2011).

V.n.: Erva-pinheira.

Ag BAl BB BL E R TM

8. Pistorinia DC.

8.1. P. hispanica (L.) DC.; Franco (1971: 247); Castroviejo (1997b: 108); Sequeira et al. (2011).

= Cotyledon hispanica L.; Coutinho (1939: 337); Sampaio (1947: 376).

V.n.: Conchelos-da-água.

BA BB TM

9. Sedum L.

9.1. S. acre L.; Coutinho (1939: 333); Sampaio (1947: 374); Franco (1971: 250); Castroviejo \& Velayos (1997a: 125); Sequeira et al. (2011).

V.n.: Uva-de-cão, vermiculária.

BA BL DL Mi TM

9.2. S. album L.; Coutinho (1939: 334); Sampaio (1947: 374); Franco (1971: 251); Castroviejo \& Velayos (1997a: 131); Smith \& Figueiredo (2010a, 2012); Sequeira et al. (2011).

V.n.: Arroz-dos-telhados, pinhões-de-rato. $\mathrm{AAl}$ Ag BA BAl BB BL DL E Mi R TM 


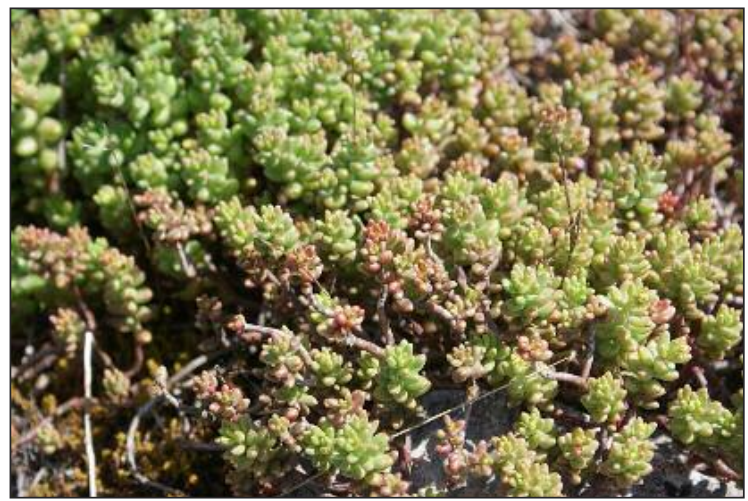

Figure 14. Close-up of the leaves of Sedum album. Photograph: Gideon F. Smith.

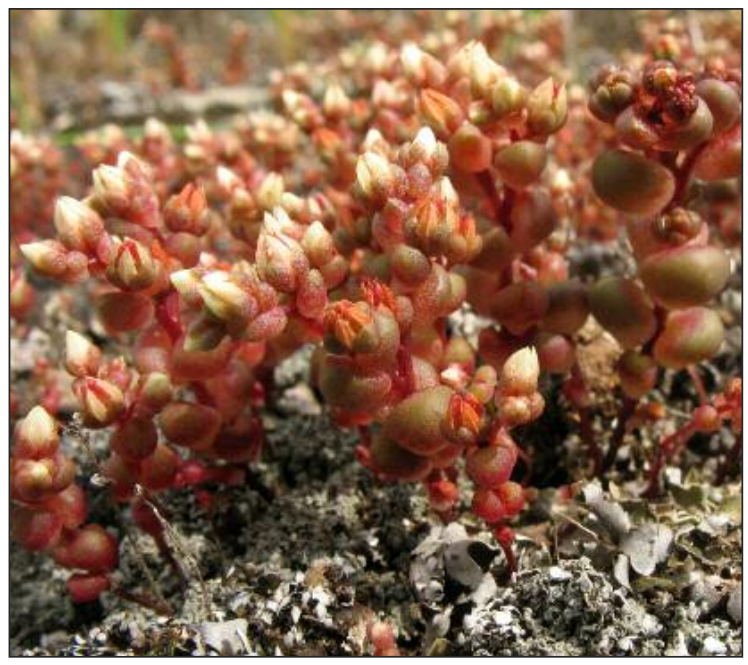

Figure 15. Sedum andegavense.

Photograph: P.P. Silva.

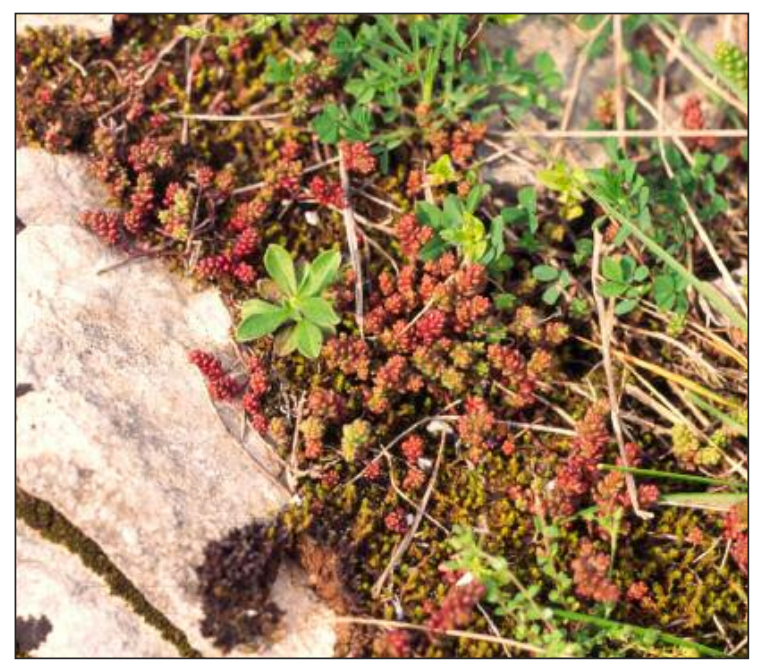

Figure 16. Sedum anglicum.

Photograph: Gideon F. Smith.

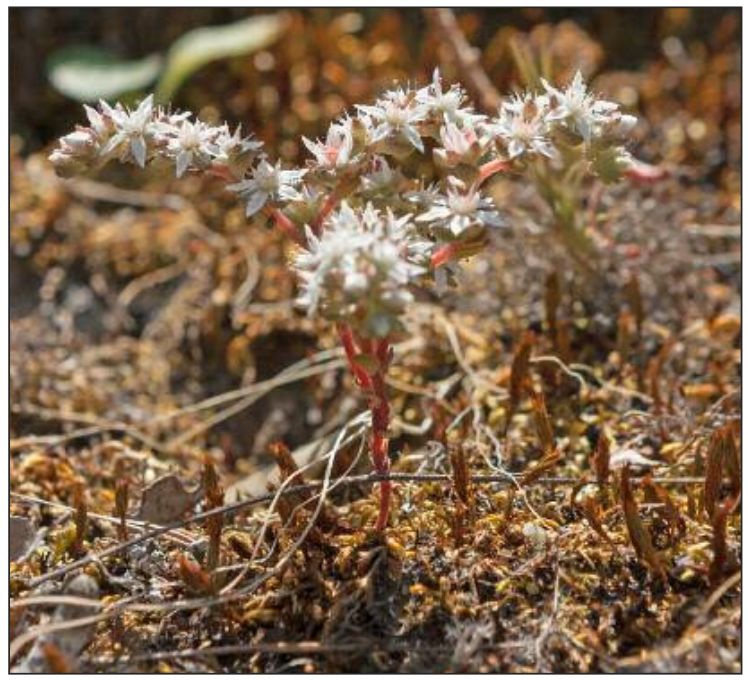

Figure 17. Sedum arenarium.

Photograph: M. Porto.

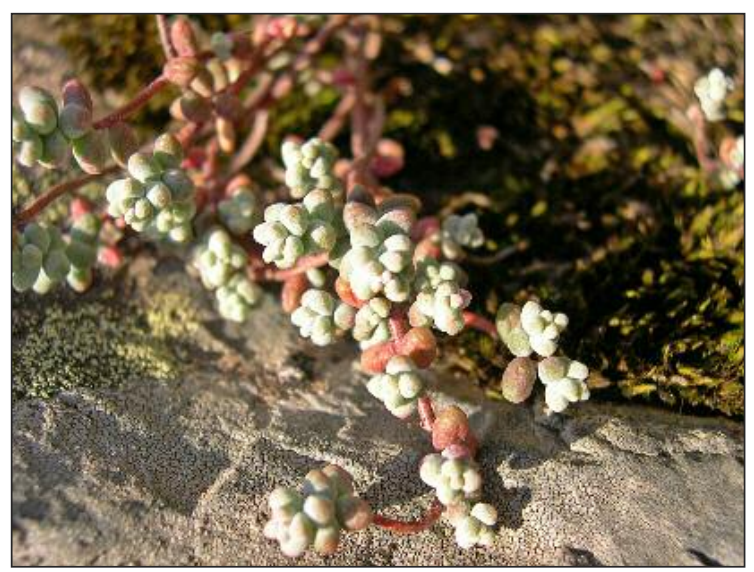

Figure 18. Sedum brevifolium.

Photograph: Hélia Marchante.

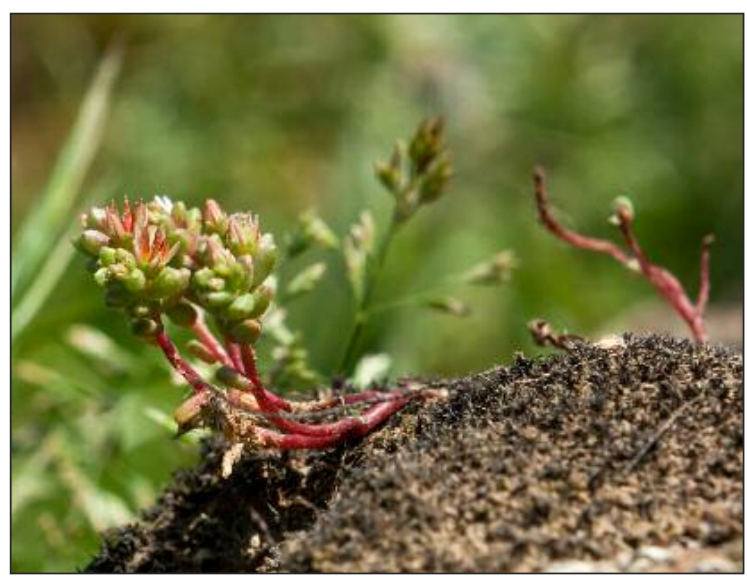

Figure 19. Sedum caespitosum.

Photograph: M. Porto. 


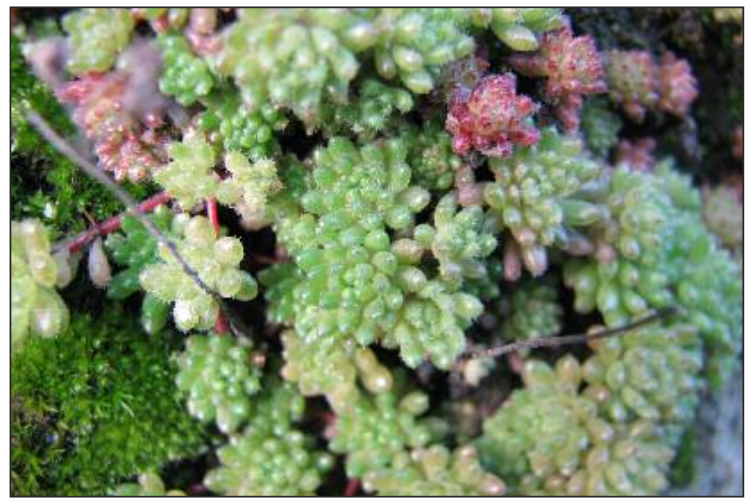

Figure 20. Sedum hirsutum.

Photograph: Hélia Marchante.

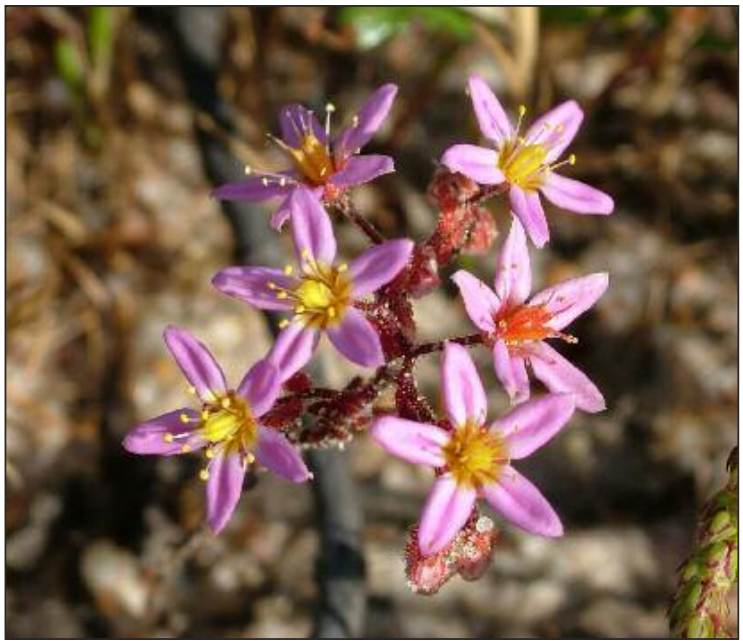

Figure 21. Sedum maireanum.

Photograph: M. Porto.

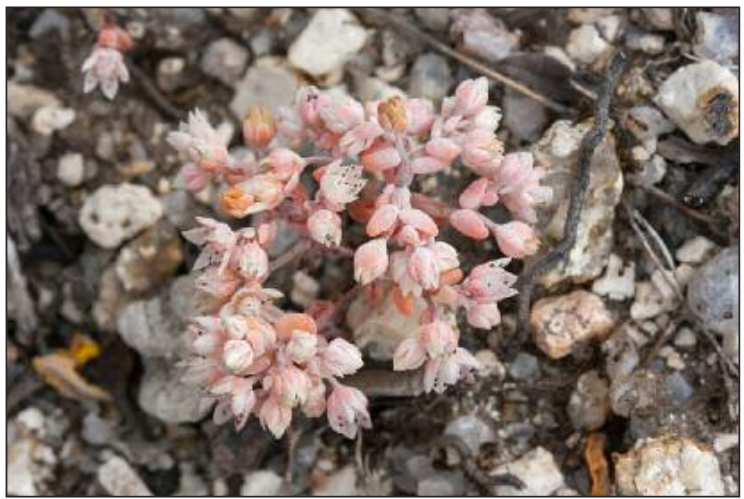

Figure 23. Sedum pedicellatum subsp. lusitanicum.

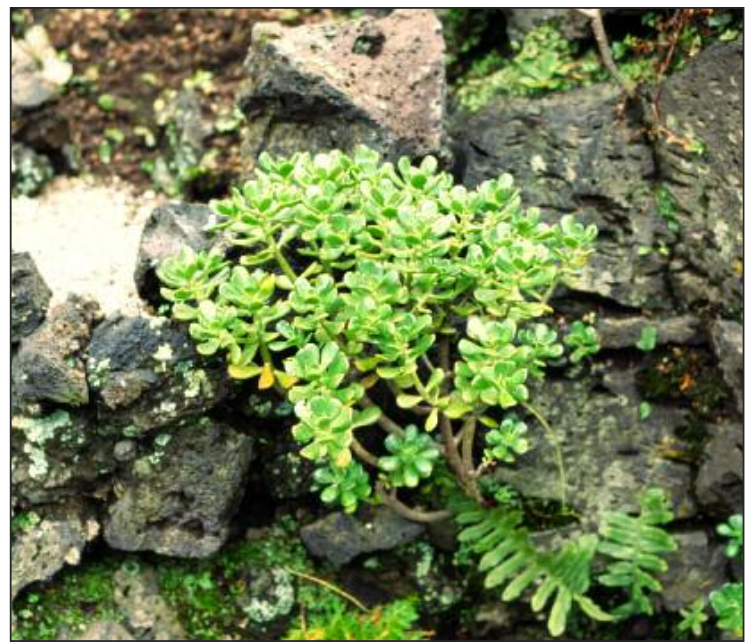

Figure 24. Shrubby growth form of * Sedum praealtum. Photograph: Gideon F. Smith.

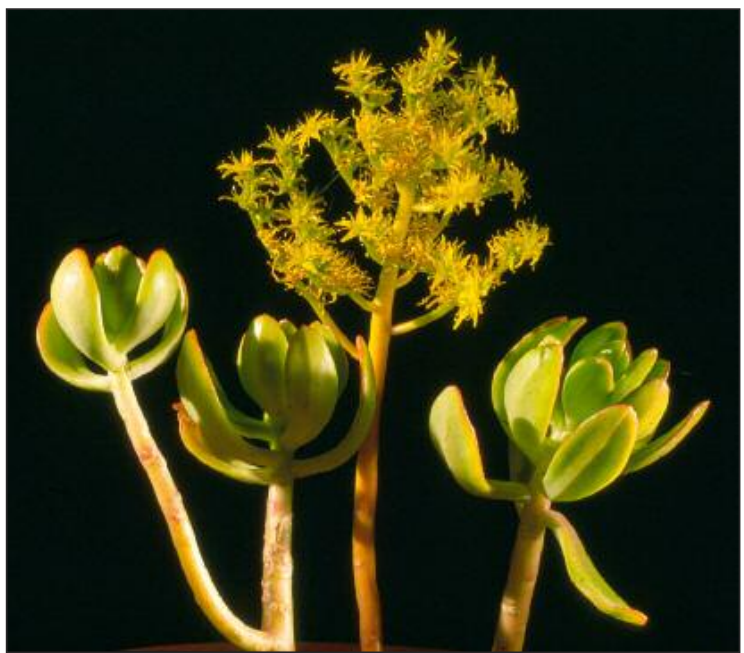

Figure 25. Close-up of leaves and an inflorescence of *Sedum praealtum.

Photograph: Gideon F. Smith. 


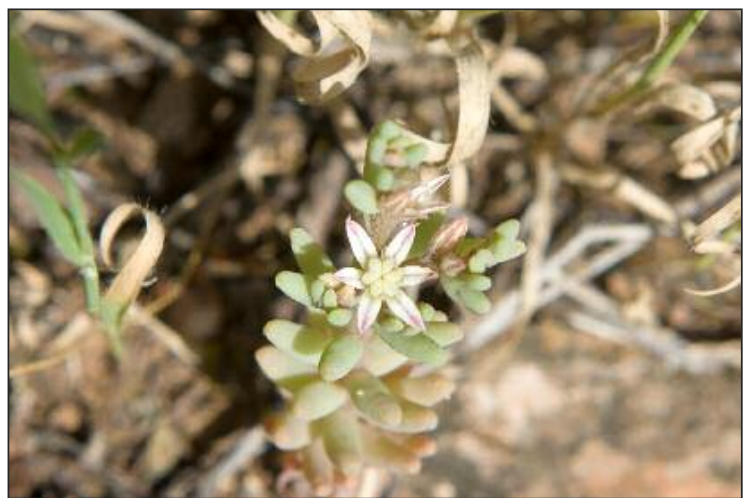

Figure 26. Sedum rubens. Photograph: M. Porto.

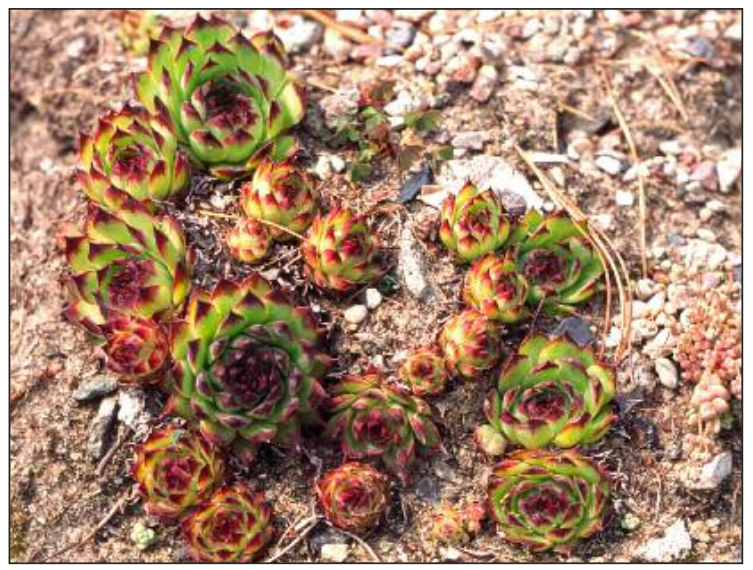

Figure 27. *Sempervivum tectorum.

Photograph: Gideon F. Smith.

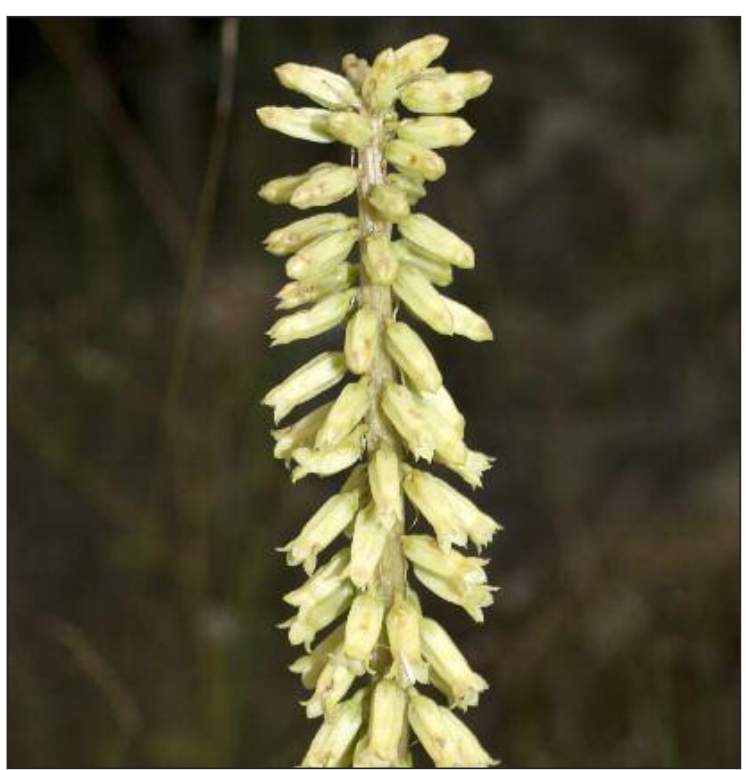

Figure 28. Umbilicus heylandianus.

Photograph: C. Aguiar.

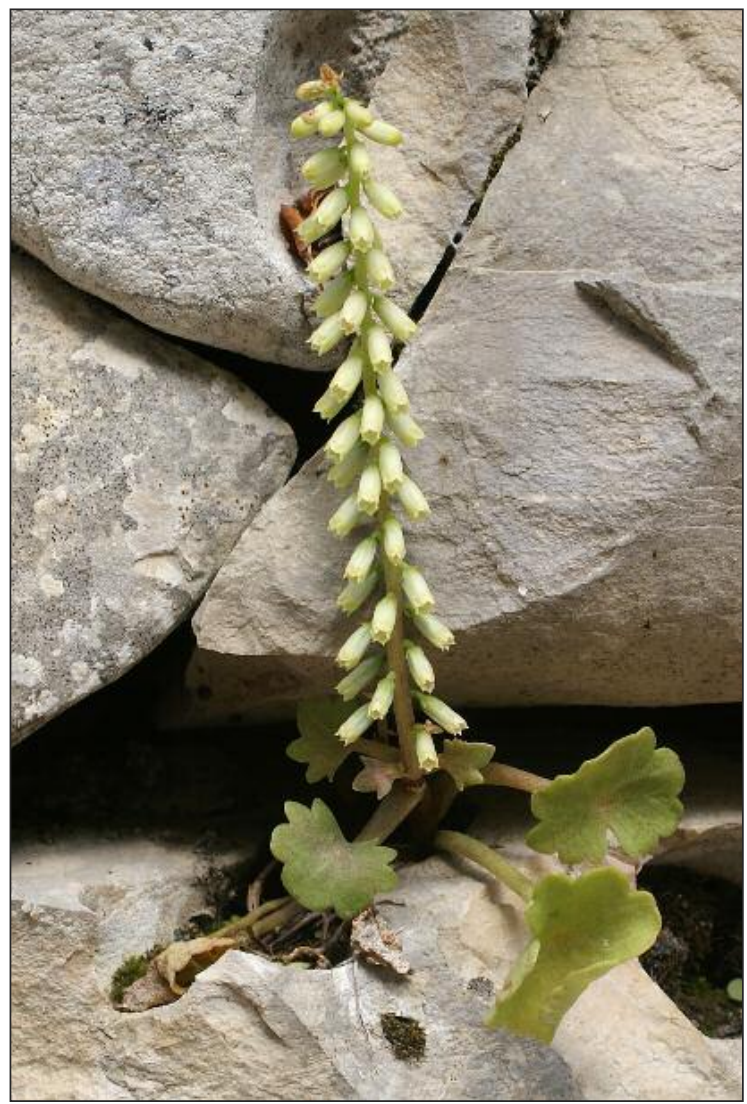

Figure 29. Umbilicus rupestris.

Photograph: Gideon F. Smith.

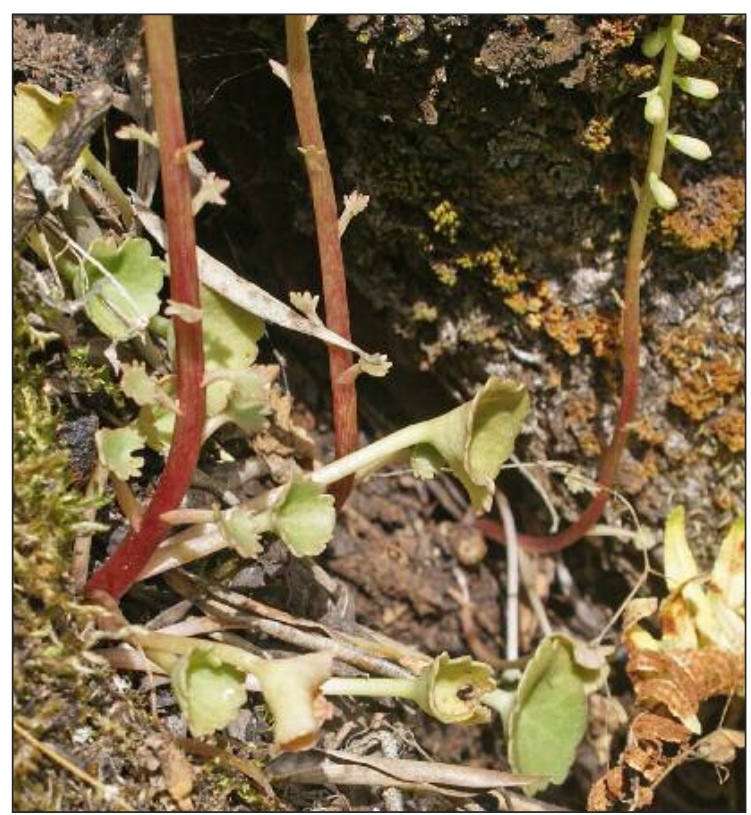

Figure 30. Close-up of the leaves of Umbilicus rupestris. Photograph: Gideon F. Smith. 
9.3. S. andegavense (DC.) Desv.; Coutinho (1939: 335); Sampaio (1947: 371); Franco (1971: 253); Castroviejo \& Velayos (1997a: 136); Sequeira et al. (2011).

AAl Ag BA BAl BB E TM

9.4. S. anglicum Huds.; Coutinho (1939: 333, pro parte); Sampaio (1947: 373, pro parte); Franco (1971: 251); Castroviejo \& Velayos (1997a: 126); Sequeira et al. (2011).

V.n.: Vermiculária-inglesa.

BA BL DL Mi TM

9.5. S. arenarium Brot.; Franco (1971: 252); Castroviejo \& Velayos (1997a: 127); Sequeira et al. (2011).

= Sedum anglicum subsp. arenarium (Brot.) Cout.; Coutinho (1939: 333).

AAl BA BB BL DL Mi R TM

9.6. S. brevifolium DC.; Coutinho (1939: 334); Sampaio (1947: 374); Franco (1971: 251); Castroviejo \& Velayos (1997a: 137); Sequeira et al. (2011).

V.n.: Arroz-dos-muros

AAl Ag BA BAl BB BL DL E Mi R TM

9.7. S. caespitosum (Cav.) DC.; Coutinho (1939: 335); Sampaio (1947: 371); Franco (1971: 253); Castroviejo \& Velayos (1997a: 142); Sequeira et al. (2011).

AAl BA E TM

9.8. S. candollei Raym.-Hamet; Castroviejo \& Velayos (1997a: 137); Sequeira et al. (2011).

= Cotyledon sediformis Lapeyr.; Sampaio (1947: 376).

= C. sedoides DC.; Coutinho (1939: 337).

= Mucizonia sedoides (DC.) D.A.Webb; Franco (1971: 248).

BA

9.9. S. hirsutum L.; Coutinho (1939: 334); Sampaio (1947: 374); Franco (1971: 251); Castroviejo \& Velayos (1997a: 135); Sequeira et al. (2011).

= Oreosedum hirsutum (All.) Grulich

$\mathrm{AAl}$ Ag BA BB BL DL Mi TM

9.10. S. maireanum Sennen; Castroviejo \& Velayos (1997a: 152); Sequeira et al. (2011).

= S. lagascae sensu auct. non Pau; Franco (1971: 252).

= S. villosum sensu auct. non L.; Sampaio (1947: 373).

AAl Ag BA BB E R TM
9.11. S. mucizonia (Ortega) Raym.-Hamet; Castroviejo \& Velayos (1997a: 135); Sequeira et al. (2011).

= Cotyledon hispida Lam., nom. illeg.; Coutinho (1939: 337).

=C. mucizonia Ortega; Sampaio (1947: 376).

= Mucizonia hispida (Lam.) A.Berger; Franco (1971: 248).

= Sedum mucizonia subsp. urceolatum R.Stephenson

$\mathrm{AAl}$ Ag BAl BB BL E R

9.12.1. S. pedicellatum Boiss. \& Reut.; Coutinho (1939: 334); Sampaio (1947: 373); Franco (1971: 252); Castroviejo \& Velayos (1997a: 139); Sequeira et al. (2011).

= Oreosedum pedicellatum (Boiss. \& Reut.) Grulich

BA BB TM

9.12.2. S. pedicellatum subsp. lusitanicum (Willk. ex Mariz) M.Laínz; Castroviejo \& Velayos (1997a: 139); Sequeira et al. (2011).

$=S$. pedicellatum var. lusitanicum Willk. ex Mariz; Coutinho (1939: 334); Sampaio (1947: 373). = S. willkommianum R.Fern.; Franco (1971: 251). BA

$9.13 *$ S. praealtum A.DC.; Almeida \& Freitas (2006: 123).

Introduced from Mexico.

9.14. S. rubens L.; Coutinho (1939: 335); Sampaio (1947: 373); Franco (1971: 252); Castroviejo \& Velayos (1997a: 130); Sequeira et al. (2011).

$\mathrm{AAl}$ Ag BA BAl BB BL DL E R TM

10. *Sempervivum L.

10.1. *S. tectorum L.; Coutinho (1939: 335); Sampaio (1947: 375); Rosseló (1997: 113); Almeida \& Freitas (2006: 127); Sequeira et al. (2011).

Recorded by Rosseló (1997) as naturalized in Mi, TM.

Introduced from Eurasia.

11. Umbilicus DC.

11.1. U. heylandianus Webb \& Berthel.; Franco (1971: 247); Castroviejo (1997c: 107); Sequeira et al. (2011).

= Cotyledon coutinhoi (Mariz) Cout.; Coutinho (1939: 336).

= C. praealta (Brot.) Samp.; Coutinho (1939: 336); Sampaio (1947: 376).

AAl BA BB DL E TM 
11.2. U. rupestris (Salisb.) Dandy; Franco (1971: 247); Castroviejo (1997c: 104); Sequeira et al. (2011); Smith \& Figueiredo (2011).

= Cotyledon neglecta Cout.; Coutinho (1939: 336).

= C. umbilicus L.; Coutinho (1939: 336); Sampaio (1947: 376).

V.n.: Cachilro, sombrerinho-dos-telhados, orelhade-monge, umbigo-de-vénus, copilas, bifes, bacelos, couxilgos, conchelos.

AAl Ag BA BAl BB BL DL E Mi R TM

\section{Acknowledgements}

Ray Stephenson has been very helpful throughout with advice and identifications. The Sociedade Portuguesa de Botânica, Carlos Aguiar, Paulo Araújo, Hélia Marchante, Miguel Porto and Patrícia Silva are thanked for kindly giving permission to use their photographs. We thank an anonymous referee for comments.

\section{References}

Aedo, C. (General Coord.) (2013). Flora iberica. http://www.floraiberica.es/, [accessed in April 2013].

Almeida, J.D. \& Freitas, H. (2006). Exotic naturalized flora of continental Portugal-A reassessment. Botanica Complutensis 30: 117-130.

Berger, A. (1930). Crassulaceae. In: EngLeR, A. \& Prantl, K. (EDS.), Die Natürlichen Pflanzenfamilien, 2nd edn. 18A: 352-483.

Castroviejo, S. (1997a). Crassulaceae. In: CAsTROVIEJo, S. ET AL. (EDS.), Flora iberica 5: 97160. Real Jardín Botánico, CSIC, Madrid.

Castroviejo, S. (1997b). Pistorinia. In: CastroVIEJO, S. ET AL. (EDS.), Flora iberica 5: 107-110. Real Jardín Botánico, CSIC, Madrid.

Castroviejo, S. (1997c). Umbilicus. In: CastroVIEJO, S. ET AL. (EDS.), Flora iberica 5: 103-107. Real Jardín Botánico, CSIC, Madrid.

Castroviejo, S. \& Velayos, M. (1997a). Sedum. In: CAstroviejo, S. et al. (EDS.), Flora iberica 5: 121-153. Real Jardín Botánico, CSIC, Madrid.

Castroviejo, S. \& Velayos, M. (1997b). Hylotelephium. In: CastrovieJo, S. ET AL. (EDS.), Flora iberica 5: 153-158. Real Jardín Botánico, CSIC, Madrid.

Coutinho, A.X.P. (1939, reprinted 1974). Familia 69. Crassuláceas. In: PALHInHA, R. (ED.), Flora de Portugal. (Plantas vasculares). Disposta em chaves dicotómicas, 2nd ed.: 332-337. Bertrand (Irmãos), Ltd, Lisboa.

Crouch, N.R. \& Smith, G.F. (2007). Crassulaceae. Bryophyllum proliferum naturalized in KwaZulu-Natal, South Africa. Bothalia 37: 206-208.
EGGLI, U. (2003b). Crassula tillaea Lester-Garland. In: Van Jaarsveld, E.J., Crassula. In: EGGLI U. (ED.), Illustrated handbook of succulent plants. Crassulaceae.: 81. Springer Verlag, Berlin.

Fernandes, R.B. (1997). Crassula. In: CastroVIEJO, S. ET AL. (EDS.), Flora iberica 5: 99-103. Real Jardín Botánico, CSIC, Madrid.

Franco, J.A. (1971). Nova Flora de Portugal (Continente e Açores), vol. 1 Lycopodiaceae-Umbelliferae. The Author, Lisboa.

Grulich, V. (1984). Generic division of Sedoideae in Europe and adjacent regions. Preslia 56: $29-54$.

Mort, M.E., Soltis, D.E., Soltis, P.S., FranciscoOrtega, J. \& Santos-Guerra, A. (2001). Phylogenetic relationships and evolution of Crassulaceae inferred from matk sequence data. American Journal of Botany 88: 76-91.

NyfFELER, R. (2003). Aichryson. In: EGGLI, U. (ED.), Illustrated handbook of succulent plants. Crassulaceae.: 24-27. Springer Verlag, Berlin.

Rosseló, J.A. (1997). Sempervivum. In: CASTRoVIEJO, S. ET AL. (EDS.), Flora iberica 5: 110-116. Real Jardín Botánico, CSIC, Madrid.

SAmpaio, G. (1947). Flora Portuguesa. 3rd facsimile edn (1988) of the 2 nd revised edn by A. Pires de Lima. Instituto Nacional de Investigação Científica, Lisboa.

Santos Guerra, A. (1997a). Aeonium. In: CastroVIEJO, S. ET AL. (EDS.), Flora iberica 5: 116-119. Real Jardín Botánico, CSIC, Madrid.

Santos Guerra, A. (1997b). Aichryson. In: CasTROVIEJO, S. ET AL. (EDS.), Flora iberica 5: 119121. Real Jardín Botánico, CSIC, Madrid.

Sequeira, M., Espírito-Santo, D., Aguiar, C. CAPElo, J. \& HonRado, J. (EDS) 2011. Checklist da flora de Portugal (continental, Açores e Madeira). http://www3.uma.pt/alfa/checklist_flora_pt.html

Sмith, G.F. \& Figueiredo, E. (2010a). Sedum album: a mainstay of European succulents. Cactus \& Succulent Journal (U.S.) 82: 41-42.

Smith, G.F. \& Figueiredo, E. (2010b). Petrosedum sediforme, a beautiful species of Crassulaceae from Portugal. CactusWorld 28: 103-106.

SMith, G.F. \& Figueiredo, E. 2011. Umbilicus rupestris: an interesting member of the Crassulaceae in Portugal. Cactus \& Succulent Journal (U.S.) 83(5):232-235.

Smith, G.F. \& Figueiredo, E. 2012. Did the Romans grow succulents in Iberia? Cactus \& Succulent Journal (U.S.) 84: 33-40.

SMith, G.F., VAn JAARsveld, E.J., ARnold, T.H., Steffens, F.E., Dixon, R.D. \& Retief, J.A. (EDS) (1997). List of southern African succulent plants. Umdaus Press, Pretoria. 
Stephenson, R. (1994). Sedum: cultivated stonecrops. Timber Press, Portland.

Stephenson, R. (2007a). An overall view of the genus Petrosedum with a description of a $P$. pruinatum location. Sedum Society Newsletter 82: 92-95.

'T HART, H. \& EGGLI, U. (EDs.) (1995). Evolution and systematics of the Crassulaceae. Backhuys Publishers, Leiden.

Thiede, J. \& EgGLI, U. (2007). Crassulaceae. In: Kubitzki, K. (ED.) The families and genera of vascular plants, vol. 9, Flowering Plants, Eudicots: 83-118. Springer Verlag, Berlin.

VAN HAM, R.C.H.J. \& 'T HART, H. (1998). Phylogenetic relationships in the Crassulaceae inferred from chloroplast DNA restriction-site variation. American Journal of Botany 85: 123-134.
VAN JAARSVELD, E.J. (2003). Crassula L. In: EGGLI, U. (ED.), Illustrated handbook of succulent plants. Crassulaceae: 32-84. Springer Verlag, Berlin.

Velayos, M. (1989). Combinaciones en Sedum s.l. (Crassulaceae). Anales Jardín Botánico de Madrid 45: 584-585.

Walters, M. (2011) Crassulaceae. In: Walters, M., Figueiredo, E., Crouch, N.R., Winter, P., Smith, G.F., Zimmermann, H.G. \& Mashope, B.K. Naturalised and invasive succulents of southern Africa: 232-259. ABC Taxa, volume 11

Webb, D.A., Favarger, C. \& Zésiger, F. (1964). Crassulaceae. In: Tutin, T.G. ET AL. (EDS.), Flora Europaea-Psilotaceae to Platanaceae, vol. 1: 350-364. Cambridge University Press, Cambridge. 